\title{
Ensemble Document Clustering Using Weighted Hypergraph Generated by NMF
}

\author{
Hiroyuki Shinnou, Minoru Sasaki \\ Ibaraki University, \\ 4-12-1 Nakanarusawa, Hitachi, \\ Ibaraki, Japan 316-8511 \\ \{shinnou,msasaki\}@mx.ibaraki.ac.jp
}

\begin{abstract}
In this paper, we propose a new ensemble document clustering method. The novelty of our method is the use of Non-negative Matrix Factorization (NMF) in the generation phase and a weighted hypergraph in the integration phase. In our experiment, we compared our method with some clustering methods. Our method achieved the best results.
\end{abstract}

\section{Introduction}

In this paper, we propose a new ensemble document clustering method using Non-negative Matrix Factorization (NMF) in the generation phase and a weighted hypergraph in the integration phase.

Document clustering is the task of dividing a document's data set into groups based on document similarity. This is the basic intelligent procedure, and is important in text mining systems (M. W. Berry, 2003). As the specific application, relevant feedback in IR, where retrieved documents are clustered, is actively researched (Hearst and Pedersen, 1996)(Kummamuru et al., 2004).

In document clustering, the document is represented as a vector, which typically uses the "bag of word" model and the TF-IDF term weight. A vector represented in this manner is highly dimensional and sparse. Thus, in document clustering, a dimensional reduction method such as PCA or SVD is applied before actual clustering (Boley et al., 1999)(Deerwester et al., 1990). Dimensional reduction maps data in a high-dimensional space into a low-dimensional space, and improves both clustering accuracy and speed.

$\mathrm{NMF}$ is a dimensional reduction method ( $\mathrm{Xu}$ et al., 2003) that is based on the "aspect model" used in the Probabilistic Latent Semantic Indexing (Hofmann, 1999). Because the axis in the reduced space by NMF corresponds to a topic, the reduced vector represents the clustering result. For a given termdocument matrix and cluster number, we can obtain the NMF result with an iterative procedure (Lee and Seung, 2000). However, this iteration does not always converge to a global optimum solution. That is, NMF results depend on the initial value. The standard countermeasure for this problem is to generate multiple clustering results by changing the initial value, and then select the best clustering result estimated by an object function. However, this selection often fails because the object function does not always measure clustering accuracy.

To overcome this problem, we use ensemble clustering, which combines multiple clustering results to obtain an accurate clustering result.

Ensemble clustering consists of generation and integration phases. The generation phase produces multiple clustering results. Many strategies have been proposed to achieve this goal, including random initialization (Fred and Jain, 2002), feature extraction based on random projection (Fern and Brodley, 2003) and the combination of sets of "weak" partitions (Topchy et al., 2003). The integration phase, as the name implies, integrates multiple clustering results to improve the accuracy of the final clustering result. This phase primarily relies on two methods. The first method constructs a new simi- 
larity matrix from multiple clustering results (Fred and Jain, 2002). The second method constructs new vectors for each instance data using multiple clustering results (Strehl and Ghosh, 2002). Both methods apply the clustering procedure to the new object to obtain the final clustering result.

Our method generates multiple clustering results by random initialization of the NMF, and integrates them with a weighted hypergraph instead of the standard hypergraph (Strehl and Ghosh, 2002). An advantage of our method is that the weighted hypergraph can be directly obtained from the NMF result.

In our experiment, we compared the k-means, NMF, the ensemble method using a standard hypergraph and the ensemble method using a weighted hypergraph. Our method achieved the best results.

\section{NMF}

The NMF decomposes the $m \times n$ term-document matrix $X$ to the $m \times k$ matrix $U$ and the transposed matrix of the $n \times k$ matrix $V$ (Xu et al., 2003), where $k$ is the number of clusters; that is,

$$
X=U V^{T} .
$$

The $i$-th document $d_{i}$ corresponds to the $i$-th row vector of $\mathrm{V}$; that is, $\boldsymbol{d}_{i}=\left(v_{i 1}, v_{i 2}, \cdots, v_{i k}\right)$. The cluster number is obtained from $\arg \max _{j \in 1: k} v_{i j}$.

For a given term-document matrix $X$, we can obtain $U$ and $V$ by the following iteration (Lee and Seung, 2000):

$$
\begin{aligned}
& u_{i j} \leftarrow u_{i j} \frac{(X V)_{i j}}{\left(U V^{T} V\right)_{i j}} \\
& v_{i j} \leftarrow v_{i j} \frac{\left(X^{T} U\right)_{i j}}{\left(V U^{T} U\right)_{i j}} .
\end{aligned}
$$

Here, $u_{i j}, v_{i j}$ and $(X)_{i j}$ represent the $i$-th row and the $j$-th column element of $U, V$ and $X$ respectively.

After each iteration, $U$ must be normalized as follows:

$$
u_{i j} \leftarrow \frac{u_{i j}}{\sqrt{\sum_{i} u_{i j}^{2}}} .
$$

Either the fixed maximum iteration number, or the distance $J$ between $X$ and $U V^{T}$ stops the iteration:

$$
J=\left\|X-U V^{T}\right\|_{F}
$$

In NMF, the clustering result depends on the initial values. Generally, we conduct NMF several times with random initialization, and then select the clustering result with the smallest value of Eq.4. The value of Eq.4 represents the NMF decomposition error and not the clustering error. Thus, we cannot alway select the best result.

\section{Ensemble clustering}

\subsection{Hypergraph data representation}

To overcome the above mentioned problem, we used ensemble clustering. Ensemble clustering consists of generation and integration phases. The first phase generates multiple clustering results with random initialization of the NMF. We integrated them with the hypergraph proposed in (Strehl and Ghosh, 2002).

Suppose that the generation phase produces $m$ clustering results, and each result has $k$ clusters. In this case, the dimension of the new vector is $\mathrm{km}$. The $(k(i-1)+c)$-th dimensional value of the data $d$ is defined as follows: If the $c$-th cluster of the $i$-th clustering result includes the data $d$, the value is 1 . Otherwise, the value is 0 . Thus, the $\mathrm{km}$ dimensional vector for the data $d$ is constructed.

Consider a simple example, where $k=3, m=4$ and the data set is $\left\{d_{1}, d_{2}, \cdots, d_{7}\right\}$. We generate four clustering results. Supposing that the first clustering result is $\left\{d_{1}, d_{2}, d_{5}\right\},\left\{d_{3}, d_{4}\right\},\left\{d_{6}, d_{7}\right\}$, we can obtain the 1st, 2nd and 3rd column of the hypergraph as follows:

$\begin{aligned} & d_{1} \\ & d_{2} \\ & d_{3} \\ & d_{4} \\ & d_{5} \\ & d_{6} \\ & d_{7}\end{aligned}\left[\begin{array}{lll}1 & 0 & 0 \\ 1 & 0 & 0 \\ 0 & 1 & 0 \\ 0 & 1 & 0 \\ 1 & 0 & 0 \\ 0 & 0 & 1 \\ 0 & 0 & 1\end{array}\right]$.

Repeating the procedure produces a total of four matrices from four clustering results. Connecting these four partial matrices, we obtain the following $7 \times 12$ matrix, which is the hypergraph.

$d_{1}$
$d_{2}$
$d_{3}$
$d_{4}$
$d_{5}$
$d_{6}$
$d_{7}$$\left[\begin{array}{llllllllllll}1 & 0 & 0 & 1 & 0 & 0 & 0 & 1 & 0 & 1 & 0 & 0 \\ 1 & 0 & 0 & 0 & 1 & 0 & 1 & 0 & 0 & 0 & 0 & 1 \\ 0 & 1 & 0 & 0 & 1 & 0 & 0 & 0 & 1 & 0 & 1 & 0 \\ 0 & 1 & 0 & 0 & 0 & 1 & 0 & 1 & 0 & 0 & 1 & 0 \\ 1 & 0 & 0 & 1 & 0 & 0 & 1 & 0 & 0 & 1 & 0 & 0 \\ 0 & 0 & 1 & 0 & 0 & 1 & 0 & 0 & 1 & 0 & 0 & 1 \\ 0 & 0 & 1 & 0 & 0 & 1 & 0 & 0 & 1 & 1 & 0 & 0\end{array}\right]$




\subsection{Weighted hypergraph vs. standard hypergraph}

Each element of the hypergraph is 0 or 1 . However, the element value must be real because it represents the membership degree for the corresponding cluster.

Fortunately, the matrix V produced by NMF describes the membership degree. Thus, we assign the real value described in $V$ to the element of the hypergraph whose value is 1 . Figure 1 shows an example of this procedure. Our method uses this weighted hypergraph, instead of a standard hypergraph for integration.

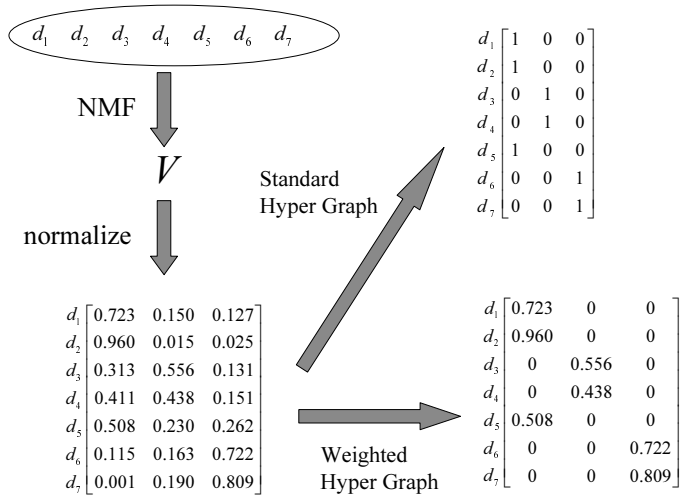

Figure 1: Weighted hypergraph through the matrix V

\section{Experiment}

To confirm the effectiveness of our method, we compared the k-means, NMF, the ensemble method using a standard hypergraph and the ensemble method using a weighted hypergraph.

In our experiment, we use 18 document data sets provided at http://glaros.dtc.umn.edu/ gkhome/cluto/cluto/download.

The document vector is not normalized for each data set. We normalize them using TF-IDF.

Table 1 shows the result of the experiment ${ }^{1}$. The value in the table represents entropy, and the smaller it is, the better the clustering result.

In NMF, we generated 20 clustering results using random initialization, and selected the cluster-

\footnotetext{
${ }^{1}$ We used the clustering toolkit CLUTO for clustering the hypergraph.
}

ing result with the smallest decomposition error. The selected clustering result is shown as "NMF" in Table 1. "NMF means" in Table 1 is the average of 20 entropy values for 20 clustering results. The "standard hypergraph" and "weighted hypergraph" in Table 1 show the results of the ensemble method obtained using the two hypergraph types. Table 1 shows the effectiveness of our method.

\section{Related works}

When we generate multiple clustering results, the number of clusters in each clustering is fixed to the number of clusters in the final clustering result. This is not a limitation of our ensemble method. Any number is available for each clustering. Experience shows that the ensemble clustering using k-means succeeds when each clustering has many clusters, and they are combined into fewer clusters, which is a heuristics that has been reported (Fred and Jain, 2002), and is available for our method

Our method uses the weighted hypergraph, which is constructed by changing the value 1 in the standard hypergraph to the corresponding real value in the matrix $V$. Taking this idea one step further, it may be good to change the value 0 in the standard hypergraph to its real value. In this case, the weighted hypergraph is constructed by only connecting multiple $V \mathrm{~s}$. We tested this complete weighted hypergraph, and the results are shown as "hypergraph V" in Table 1.

"Hypergraph V" was better than the standard hypergraph, but worse than our method. Furthermore, the value 0 may be useful because we can use the graph spectrum clustering method (Ding et al., 2001), which is a powerful clustering method for the spare hypergraph.

In clustering, the cluster label is unassigned. However, if cluster labeling is possible, we can use many techniques in the ensemble learning (Breiman, 1996). Cluster labeling is not difficult when there are two or three clusters. We plan to study this approach of the labeling cluster first and then using the techniques from ensemble learning.

\section{Conclusion}

This paper proposed a new ensemble document clustering method. The novelty of our method is the use 
Table 1: Document data sets and Experiment results

\begin{tabular}{|l|c|c|c||c|c|c|c|c||c|}
\hline Data & $\begin{array}{c}\text { \# of } \\
\text { doc. }\end{array}$ & $\begin{array}{c}\text { \# of } \\
\text { terms }\end{array}$ & $\begin{array}{c}\text { \# of } \\
\text { classes }\end{array}$ & k-means & NMF & $\begin{array}{c}\text { NMF } \\
\text { means }\end{array}$ & $\begin{array}{c}\text { Standard } \\
\text { hypergraph }\end{array}$ & $\begin{array}{c}\text { Weighted } \\
\text { hypergraph }\end{array}$ & $\begin{array}{c}\text { Hypergraph } \\
\text { V }\end{array}$ \\
\hline cacmcisi & 4663 & 41681 & 2 & 0.750 & 0.817 & 0.693 & 0.691 & $\mathbf{0 . 6 9 0}$ & 0.778 \\
cranmed & 2431 & 41681 & 2 & $\underline{\mathbf{0 . 1 1 3}}$ & 0.963 & 0.792 & 0.750 & 0.450 & 0.525 \\
fbis & 2463 & 2000 & 17 & 0.610 & 0.393 & 0.406 & 0.408 & $\mathbf{0 . 3 8 1}$ & 0.402 \\
hitech & 2301 & 126373 & 6 & $\underline{\mathbf{0 . 5 8 5}}$ & 0.679 & 0.705 & 0.683 & 0.684 & 0.688 \\
k1a & 2340 & 21839 & 20 & 0.374 & 0.393 & 0.377 & 0.386 & $\mathbf{0 . 3 5 1}$ & 0.366 \\
k1b & 2340 & 21839 & 6 & 0.221 & 0.259 & 0.238 & 0.456 & 0.216 & $\underline{\mathbf{0 . 2 0 5}}$ \\
la1 & 3204 & 31472 & 6 & 0.641 & 0.464 & 0.515 & $\mathbf{0 . 4 5 8}$ & 0.459 & 0.491 \\
la2 & 3075 & 31472 & 6 & 0.620 & 0.576 & 0.551 & 0.548 & $\underline{\mathbf{0 . 4 6 8}}$ & 0.486 \\
re0 & 1504 & 2886 & 13 & $\mathbf{0 . 3 6 8}$ & 0.419 & 0.401 & 0.383 & 0.379 & 0.378 \\
re1 & 1657 & 3758 & 25 & 0.374 & 0.364 & 0.346 & 0.334 & $\mathbf{0 . 3 2 5}$ & 0.337 \\
reviews & 4069 & 126373 & 5 & $\mathbf{0 . 3 6 4}$ & 0.398 & 0.538 & 0.416 & 0.408 & 0.391 \\
tr11 & 414 & 6429 & 9 & 0.349 & 0.338 & 0.311 & 0.300 & 0.304 & $\mathbf{0 . 2 8 0}$ \\
tr12 & 313 & 5804 & 8 & 0.493 & 0.332 & 0.375 & 0.308 & $\mathbf{0 . 3 0 7}$ & 0.316 \\
tr23 & 204 & 5832 & 6 & 0.527 & 0.485 & 0.489 & 0.493 & 0.521 & $\mathbf{0 . 4 7 4}$ \\
tr31 & 927 & 10128 & 7 & 0.385 & 0.402 & 0.383 & 0.343 & 0.334 & $\underline{\mathbf{0 . 3 1 0}}$ \\
tr41 & 878 & 7454 & 10 & 0.277 & 0.358 & 0.299 & $\mathbf{0 . 2 4 5}$ & 0.270 & 0.340 \\
tr45 & 690 & 8261 & 10 & 0.397 & 0.345 & 0.328 & 0.277 & $\mathbf{0 . 2 7 4}$ & 0.380 \\
wap & 1560 & 6460 & 20 & 0.408 & 0.371 & 0.374 & 0.336 & $\underline{\mathbf{0 . 3 2 7}}$ & 0.344 \\
\hline Average & 1946.2 & 27874.5 & 9.9 & 0.436 & 0.464 & 0.451 & 0.434 & $\underline{\mathbf{0 . 3 9 7}}$ & 0.416 \\
\hline
\end{tabular}

of NMF in the generation phase and a weighted hypergraph in the integration phase. One advantage of our method is that the weighted hypergraph can be obtained directly from the NMF results. Our experiment showed the effectiveness of our method using 18 document data sets. In the future, we will use an ensemble learning technique by labeling clusters.

\section{References}

D. Boley, M. L. Gini, R. Gross, E. Han, K. Hastings, G. Karypis, V. Kumar, B. Mobasher, and J. Moore. 1999. Document categorization and query generation on the world wide web using webace. Artificial Intelligence Review, 13(5-6):365-391.

L. Breiman. 1996. Bagging predictors. Machine Learning, 24(2):123-140.

S. C. Deerwester, S. T. Dumais, T. K. Landauer, G. W. Furnas, and R. A. Harshman. 1990. Indexing by latent semantic analysis. Journal of the American Society of Information Science, 41(6):391-407.

C. Ding, X. He, H. Zha, M. Gu, and H. Simon. 2001. Spectral Min-max Cut for Graph Partitioning and Data Clustering. In Lawrence Berkeley National Lab. Tech. report 47848 .

X. Z. Fern and C. E. Brodley. 2003. Random Projection for High Dimensional Data Clustering: A Cluster Ensemble Approach. In the 20th International Conference of Machine Learning (ICML-03).
A.L.N. Fred and A. K. Jain. 2002. Data Clustering Using Evidence Accumulation. In the 16th international conference on pattern recognition, pages 276-280.

M. A. Hearst and J. O. Pedersen. 1996. Reexamining the cluster hypothesis: Scatter/gather on retrieval results. In Proceedings of SIGIR-96, pages 76-84.

T. Hofmann. 1999. Probabilistic Latent Semantic Indexing. In Proceedings of the 22nd Annual ACM Conference on Research and Development in Information Retrieval, pages 50-57.

K. Kummamuru, R. Lotlikar, S. Roy, K. Singal, and R. Krishnapuram. 2004. A Hierarchical Monothetic Document Clustering Algorithm for Summarization and Browsing Search Results. In Proceedings of $W W W-04$, pages 658-665.

D. D. Lee and H. S. Seung. 2000. Algorithms for nonnegative matrix factorization. In NIPS, pages 556562.

M. W. Berry, editor. 2003. Survey of Text Mining: Clustering, Classification, and Retrieval. Springer.

A. Strehl and J. Ghosh. 2002. Cluster Ensembles - A Knowledge Reuse Framework for Combining Multiple Partitions. In Conference on Artificial Intelligence (AAAI-2002), pages 93-98.

A. Topchy, A. K. Jain, and W. Punch. 2003. Combining Multiple Weak Clusterings.

W. Xu, X. Liu, and Y. Gong. 2003. Document clustering based on non-negative matrix factorization. In Proceedings of SIGIR-03, pages 267-273. 